\title{
Towards Component-based, Domain-specific, Efficient Question Answering Systems
}

\author{
Andreas Both \\ DATEV eG \\ DATEV eG, 90329 Nürnberg, Germany \\ Andreas.Both@datev.de
}

\begin{abstract}
In the past, the research, as well as industry, brought Question Answering (QA) into daily use. However, there is still an obvious gap between the claim of providing access to any - structured or unstructured - knowledge stored in the world using an interface fitting the demands of regular users. On the one hand side, implementing Question Answering Systems is still hard and time-consuming, on the other hand side, the QA community is still struggling on defining a common ground for collaboration across research fields regarding, for example, realistic benchmarks, maintainability, and broad coverage of knowledge sources.

In the talk, challenges of Question Answering will be highlight w.r.t. hybrid QA, domain-specific QA, cross knowledge base QA, etc. Particularly the industry perspective is also presented while aiming at a Question Answering platform which can be assembled from industry components as well as components of the research community. First steps towards this long-term vision are provided by the Qanary framework and similar frameworks aiming at a collaborative approach for the development QA systems which should lead to effective implementations, improved research results as well as a platform economy for QA. Such a platform would lead industry to a tighter collaboration while academics would have the opportunity of accessing precious data for further improvements.
\end{abstract}

\section{KEYWORDS}

Question-Answering, hybrid QA, domain-specific QA systems, QA for industry

\section{ACM Reference Format:}

Andreas Both. 2018. Towards Component-based, Domain-specific, Efficient Question Answering Systems. In WWW'18 Companion: The 2018 Web Conference Companion, April 23-27, 2018, Lyon, France. ACM, New York, NY, USA, 1 page. https://doi.org/10.1145/3184558.3193132
This paper is published under the Creative Commons Attribution 4.0 International (CC BY 4.0) license. Authors reserve their rights to disseminate the work on their personal and corporate Web sites with the appropriate attribution.

WWW'18 Companion, April 23-27, 2018, Lyon, France

() 2018 IW3C2 (International World Wide Web Conference Committee), published under Creative Commons CC BY 4.0 License.

ACM ISBN 978-1-4503-5640-4/18/04.

https://doi.org/10.1145/3184558.3193132

\section{BIOGRAPHY}

Dr. Andreas Both is a computer scientist dedicated to applied research in an industrial context, in particular, data-driven processes, data integration, information retrieval applications and web engineering. Currently, he is Head of Architecture, Web Technologies and IT Research at DATEV eG a German company dedicated to business software. He commits himself to advance in using the World Wide Web (WWW) as knowledge base and developing the next generation of Web applications to open the capabilities of the WWW for both industry and users. Since June 2016 Andreas Both is active as member of the WDAqua advisory board. 